\title{
Sistema Especialista Web para Análise de Alimentos
}

\author{
Adriana Soares Pereira, André Paulo Sartori, Jacson Luis Krotz \\ Universidade Federal de Santa Maria (UFSM) \\ Caixa Postal 54 - 98.400-000 - Frederico Westphalen - RS - Brazil \\ adriana.pereira@ufsm.br, andresartori3@gmail.com, luis.kr@live.com
}

\begin{abstract}
Resumo. Este trabalho fez parte do projeto de desenvolvimento do Curso Técnico em Agroindústria, da Universidade Federal de Santa Maria, na modalidade a distância. Um dos grandes desafios deste curso foi o desenvolvimento de conteúdos por parte do professor. Para auxiliar os professores em muitas disciplinas do curso, este trabalho teve por objetivo desenvolver um sistema especialista web para análise de alimentos (ANALI). Entre as funções do protótipo estão o auxílio a problemas relacionados à área de nutrição como a obesidade, a má nutrição além de distúrbios alimentares. $O$ desenvolvimento do sistema, bem como informações relacionadas à pesquisa em questão são abordadas no decorrer deste trabalho.
\end{abstract}

\section{Introdução}

Assim como a tecnologia evolui nos dias atuais, problemas relacionados a má alimentação aumentam gradativamente. Inúmeros são os casos de obesidade infantil, doenças causadas pela má alimentação e também a falta de uma orientação direcionada a práticas saudáveis [Tomkins, 2006]. Neste contexto, sistemas especialistas podem contribuir na solução de problemas relacionados à área de nutrição, indicando e evidenciando uma prática saudável e nutricional aos usuários. Estes por sua vez, podem obter grandes benefícios ao contar com o auxílio tecnológico para a sua solução de problemas relacionados a nutrição em seu dia-a-dia. Observa-se de modo geral, que problemas de obesidade tem crescido bastante na sociedade contemporânea e problemas de escala inversa (como por exemplo, a má nutrição) também apresentam dados significativos. Aliando estes dados ao uso de ferramentas tecnológicas é possível aprofundar o estudo de soluções para o tratamento de distúrbios alimentares.

Um exemplo deste cenário é o Brasil, um país com um nível de desenvolvimento exponencial, porém com problemas que antes afligiam apenas países desenvolvidos, como a crescente crise de obesidade e a demanda por soluções para o auxílio em nutrição esportiva e manutenção de um estilo de vida saudável. O Brasil também por anos luta contra problemas como a subnutrição, principalmente a subnutrição infantil, onde por meio de soluções tecnológicas possa se obter meios mais eficientes para auxiliar na solução deste problema, objetivo proposto por este trabalho. Estes dados apontados podem ser comprovados por relatórios da ONU [Hunger, 2014] sobre a fome 
V Congresso Brasileiro de Informática na Educação (CBIE 2016)

Anais dos Workshops do V Congresso Brasileiro de Informática na Educação (CBIE 2016)

no mundo e também com as estatísticas do IBGE sobre a crescente crise de obesidade no Brasil [IBGE, 2010].

Desta forma, este trabalho apresenta um sistema especialista web, voltado a análise de alimentos, o qual foi desenvolvido para ser de fácil utilização e auxílio na aprendizagem dos alunos do Curso de Agroindústria na modalidade à distância, ofertado pela Universidade Federal de Santa Maria. Este trabalho será disponibilizado para o público em geral, por tratar-se de uma pesquisa pública e que deve estar ao alcance de toda a comunidade acadêmica.

\section{Apresentação do Software}

Com a utilização das linguagens de programação para web PHP [Achour, 2013] e linguagem de acesso a banco de dados SQL [MYSQL, 2013], foi realizado o desenvolvimento do protótipo denominado ANALI (sistema especialista web para análise de alimentos). Através do sistema especialista web [Py, 2013] são executadas as consultas e a gravação dos dados no banco.

É possível também através do sistema recuperar dados, processar e armazenar os resultados com uma série de consultas. A cada nova consulta a ideia é gerar novos dados sobre as consultas executadas. O projeto, por meio do uso do conceito de MVC (Model, View, Control) e orientação a objetos possibilita a reutilização dos códigos desenvolvidos para um ganho de agilidade no processo de programação e desenvolvimento da ferramenta.

O projeto foi desenvolvido em modelo Open-Source com o intuito de que os códigos permaneçam disponíveis para consulta durante e após o desenvolvimento, assim será utilizada a plataforma Github [Chacon, 2013] para a disponibilização dos scripts e ainda controle de versionamento do projeto.

Como amostra da ferramenta, na realização das primeiras análises de dados codifica-se um script que realiza a inserção dos dados da Tabela Brasileira de Composição de Alimentos (TACO) na base de dados modelada, utilizando o padrão criado para o projeto com base no cabeçalho da Tabela Brasileira de Composição de Alimentos e sua organização onde os caracteres textuais são substituídos por caracteres numéricos como pode-se observar na tabela 1.

A partir da utilização deste padrão e a disponibilidade do projeto na plataforma Github, permite-se que o projeto evolua constantemente sem adição de custos e também gerando maior probabilidade de atualização das versões do projeto bem como aumento da quantidade e qualidade de dados para o desenvolvimento em código aberto. 
V Congresso Brasileiro de Informática na Educação (CBIE 2016)

Anais dos Workshops do V Congresso Brasileiro de Informática na Educação (CBIE 2016)

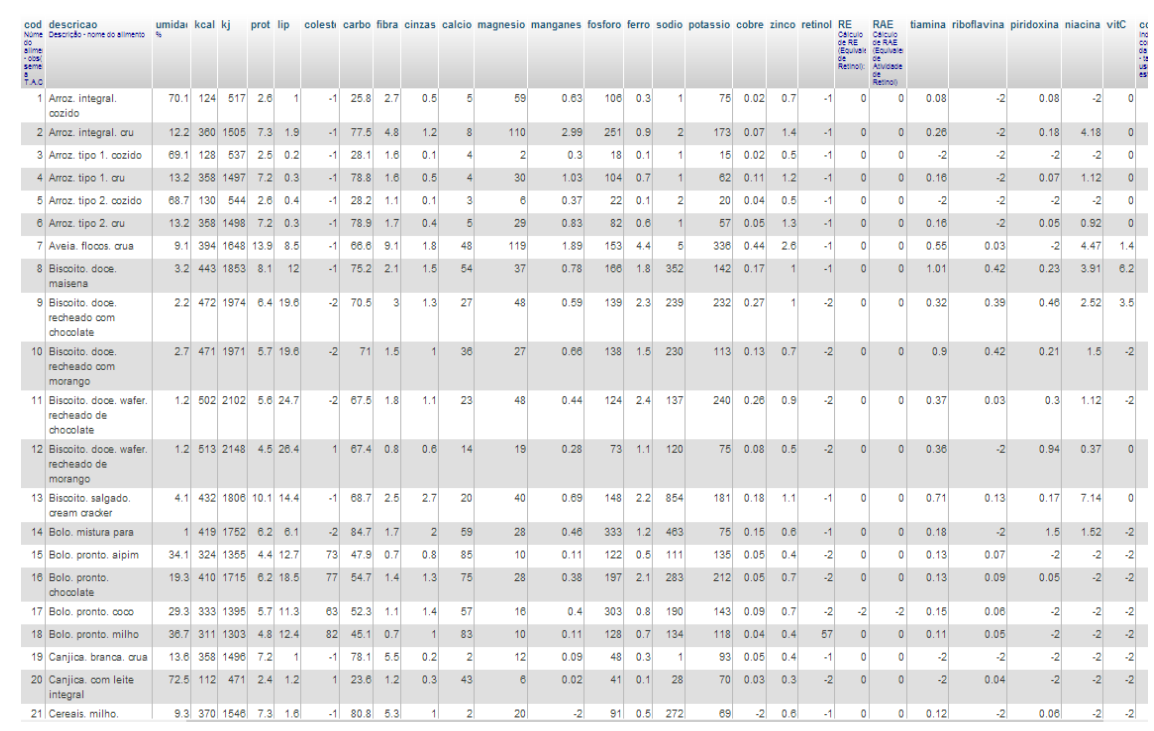

Tabela 1. Parte da tabela TACO normatizada para o uso neste trabalho

\section{1 - Desenvolvimento do Sistema}

O processo de criação de um sistema especialista é longo e deve reunir conhecimentos em diversas áreas para que se possa alcançar o resultado desejado. Demonstra-se nesta seção os resultados alcançados pelo desenvolvimento. As camadas de dados e controle demonstram scripts com codificação, então esta seção deve concentrar-se mais nos resultados demonstrados por meio da interação destas com as camadas de dados e controle.

O acesso ao sistema desenvolvido inicia-se em uma tela de Login, nesta tela, o usuário entra com o seu nome e senha, assim se for um administrador, ele é direcionado para página Inicial (Index), com privilégios administrativos, senão é direcionado a uma página sem os mesmos. A figura 1 apresenta a tela inicial.

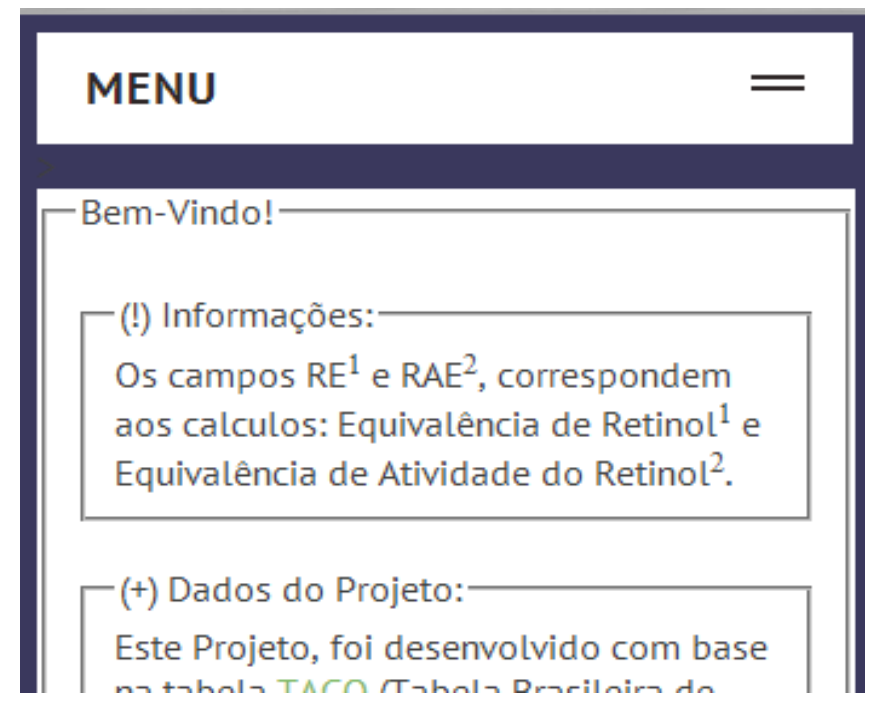

Figura 1. Tela inicial do Sistema Especialista em Análise de Alimentos 
V Congresso Brasileiro de Informática na Educação (CBIE 2016)

Anais dos Workshops do V Congresso Brasileiro de Informática na Educação (CBIE 2016)

O sistema possui uma tela de menu, onde na opção alimentos, ele é levado a tela de pesquisa, nesta tela o usuário seleciona o item que deseja pesquisar, ele pode optar pesquisar de diversas formas.

$\mathrm{Na}$ primeira forma, deixando o campo de pesquisa sem nenhum valor, são exibidos todos os valores da base de dados, selecionando-se descrição e escrevendo parte do nome de um alimento, todos os alimentos relacionados ao termo pesquisados são mostrados na tela.

O usuário ainda conta com a opção de pesquisa por código, onde este código retorna apenas o elemento de código igual ao código pesquisado. Das propriedades de pesquisa apenas a descrição e o código são obrigatórios, o usuário pode utilizar o botão selecionar todas para que todas as propriedades sejam mostradas na tela, ou selecionar uma a uma, além da pesquisa de alimentos, também pode-se realizar a pesquisa por aminoácidos, e a pesquisa por lipídeos, onde para aminoácidos e lipídeos, apenas os administradores podem visualizar estas pesquisas sem visualizar juntamente as informações de da tabela alimentos. Ainda pode-se cadastrar, alterar e excluir dados, e inserir um IDR sendo administrador, o alterar o mesmo, o IDR é obrigatório para que os resultados sejam calculados. A figura 2 demonstra a tela de pesquisas do sistema especialista.

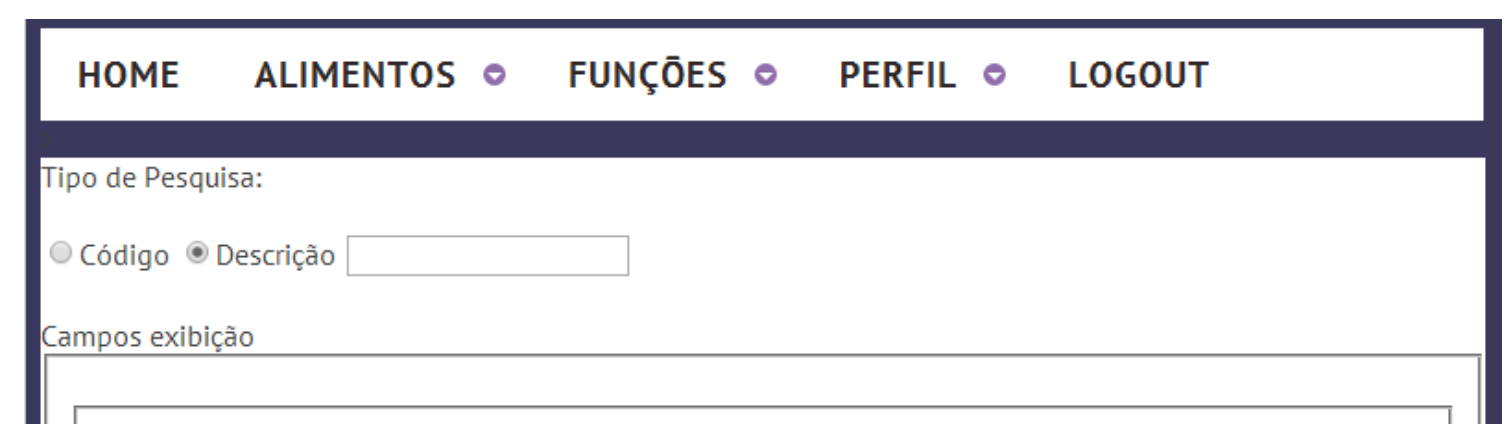

Figura 2. Tela de pesquisas (Alimentos)

A figura 3 mostra a tela de visualização da grade alimentar. Quando os resultados são mostrados, pode-se selecionar o alimento e adiciona-lo a grade alimentar, após se pode consultar a grade e remover os alimentos indesejados, nela também confere-se os valores diários recomendados e os valores gerados pelo sistema. 
V Congresso Brasileiro de Informática na Educação (CBIE 2016)

Anais dos Workshops do V Congresso Brasileiro de Informática na Educação (CBIE 2016)

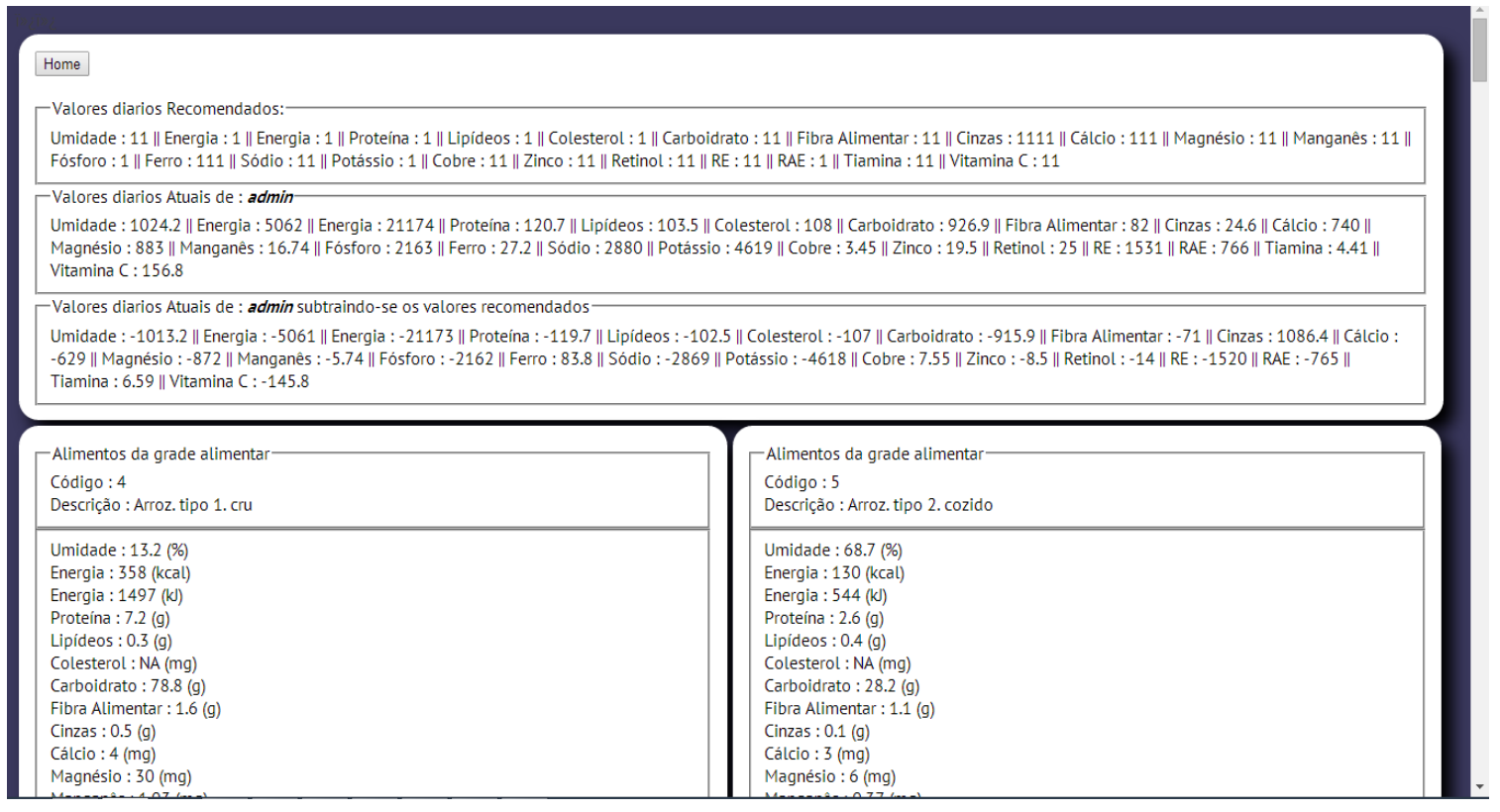

Figura 3. Tela Parcial de Resultados

O sistema também colabora com a análise da linguagem, onde não se encontra a correção para a sobrecarga de métodos naturalmente.

O uso da plataforma Github, ainda permite a utilização de padrões utilizados pelos usuários da plataforma, auxiliando o projeto a tornar-se extensível e de código aberto, compatível com dispositivos móveis e multiplataforma por meio de um navegador.

O sistema também disponibiliza de uma interface compatível com dispositivos móveis, por meio do uso de layouts fluidos e da divisão do conteúdo em scripts componentes em forma de vários módulos, este permite a extensibilidade do layout a muitas outras aplicações, sem grandes perdas, beneficiando-se do uso deste layout pôr o mesmo ser redimensionável ou seja, quando o navegador é redimensionado, ele se adapta e não cria barras de rolagem verticais que atrapalham a visualização do conteúdo. O layout também pode ser usado em dispositivos móveis, visto que o menu se adapta a eles como é visto na figura 4, que mostra o menu expandido, onde o usuário clica na opção desejada e após clica novamente, e é exibido o menu correspondente. 
V Congresso Brasileiro de Informática na Educação (CBIE 2016)

Anais dos Workshops do V Congresso Brasileiro de Informática na Educação (CBIE 2016)
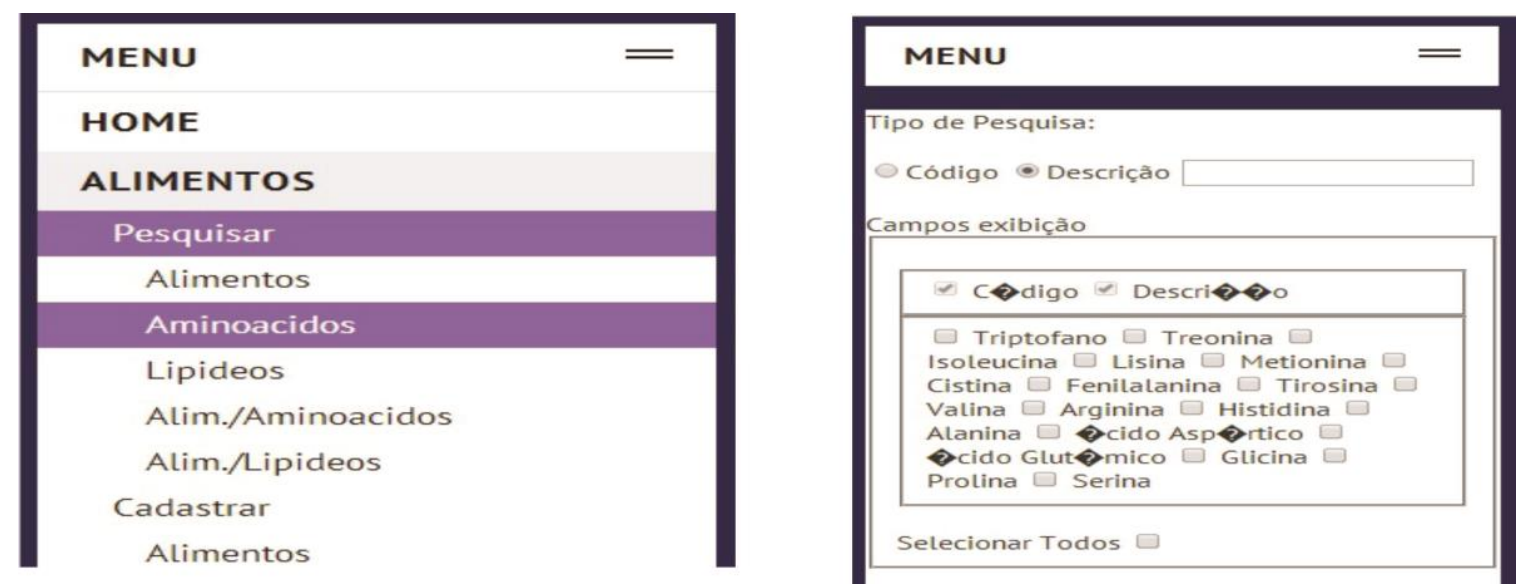

Figura 4. Interface adaptável aos dispositivos móveis, menu expandido, e resultado do menu selecionado (visualização parcial)

\section{2 - Validação e Integração do Software no Ambiente Virtual Moodle}

O protótipo desenvolvido foi validado e testado pelos professores do Curso Técnico em Agroindústria $\mathrm{EaD}$. Após essa validação, a próxima etapa deste trabalho será a integração do sistema no Ambiente Virtual Moodle, o qual é utilizado como ferramenta de ensino-aprendizagem do curso.

Com a disponibilização deste sistema, como um apoio a diversas disciplinas da grade curricular do Curso Técnico em Agroindústria, acredita-se que o aluno poderá ter uma melhor compreensão destes conteúdos.

\section{Considerações finais}

Por meio da programação e da análise da tabela TACO, chegou-se à criação de um Sistema Especialista em Análise de Alimentos. No sistema, o administrador inicia a sua utilização, adicionando os valores dos Índices Diários Recomendados, tanto para alimentos, quanto para Aminoácidos e Lipídeos. Pensa-se que deste modo, por não definir os IDRs em uma tabela, tal qual é realizado com os alimentos, garante-se que o sistema não fique atrelado apenas a uma única função. O sistema ainda pode se estender a outros fins, como a situação hipotética onde uma nutricionista ou um pesquisador responsável, entram com os valores dos IDRs, e os usuários, por meio do sistema, estes tendo menos permissões e não realizando alterações no IDR, acompanham a sua grade alimentar, e verificam os valores necessários de algum nutriente.

O conhecimento especialista concentra-se nas bases adicionadas inicialmente ao sistema, onde a partir destas se geram as grades alimentares. O sistema vem como um auxiliar, e nunca como substituto aos conhecimentos humanos, esta informação revelase importante, já que o mesmo é criado para ser uma ferramenta e não um meio de obtenção de resultados apenas. 
V Congresso Brasileiro de Informática na Educação (CBIE 2016)

Anais dos Workshops do V Congresso Brasileiro de Informática na Educação (CBIE 2016)

Cada vez mais, softwares especialistas vem sendo usados, como ferramentas de auxílio em muitas áreas. O desenvolvimento de um software especialista com o uso da linguagem PHP, provou-se possível, viável, prático e de maneira geral contribui para a expansão da internet como veículo de aquisição de soluções.

A área de alimentos pode contar com mais uma ferramenta de auxílio, e com esta para a criação de novas ferramentas, a partir desta, ou das ideias expostas neste trabalho, o trabalho multidisciplinar, faz com que a tecnologia possa se inserir em diversas áreas auxiliando a realização de tarefas e melhorando produtividade, delegando tarefas repetitivas a maquinas, trazendo foco em questões humanas a especialistas humanos.

O projeto poderá ser utilizado como um auxílio para nutricionistas, por exemplo, onde cada pessoa poderá contar com o sistema durante vinte e quatro horas para auxiliar em determinadas situações nutricionais, como: dietas hipocalóricas, hipercalóricas, na consulta de valores de alimentos, ou mesmo em dietas de suplementação vitamínica, oferecendo também alimentos de maior qualidade mesmo para usuários sem conhecimentos da área de nutrição que estarão sendo assistidos. O sistema permitirá maior agilidade nos processos de auxiliando pesquisadores e interessados facilitando as análises e a disposição das informações em diversas áreas, pesquisas e projetos.

Como proposta futura pretende-se avaliar o uso do sistema integrado ao Ambiente Virtual Moodle, plataforma esta utilizada no projeto dos cursos técnicos na modalidade EaD.

\section{Referências}

Achour. Mehdi, Betz. Friedhelm, Dovgal Antony, Lopes Nuno, Magnusson. Hannes, Richter. Georg, Seguy. Damien, Vrana. Jakub, Vrana ,2013. Manual do php. Disponível em: http://www.php.net/manual/pt_BR/. Acesso em: 29 de Julho de 2013.

Chacon, Scott .2009. Pro Git. Disponível em: https://github.s3.amazonaws.com/media/progit.en.pdf. Acesso em: 29 de Julho de 2013.

Hunger Map. World Food Programe, 2012. Disponível em: http://documents.wfp.org/stellent/groups/public/documents/communications/wfp229 327.pdf; Acesso em: 29 de Julho de 2014.

IBGE, 2010. Malnutrition decreases and weight of brazilian children surpasses international standards. Disponível em: http://saladeimprensa.ibge.gov.br/en/noticias?view=noticia\&id=1\&busca=1\&idnotici $\mathrm{a}=1699$. Acesso em: 29 de Julho de 2013.

MYSQL Reference Manual. Disponível em: http://dev.mysql.com/doc/refman/5.6/en/index.html. Acesso em: 29 de Julho de 2013.

NEPA - Núcleo de Estudos e Pesquisas em Alimentação - Universidade Estadual de Campinas - UNICAMP, tabela brasileira de composição de alimentos - taco (Segunda Edição), 2014. 
V Congresso Brasileiro de Informática na Educação (CBIE 2016)

Anais dos Workshops do V Congresso Brasileiro de Informática na Educação (CBIE 2016)

Paintr, J. , 2000, University of Illinois in the Department of Food Science and Human Nutrition., Nutrition Analysis Tool 2.0.

Py, Mônica Xavier - Instituto de Informática Universidade Federal do Rio Grande do Sul - Sistemas Especialistas: uma introdução, 2013.

Tomkins, Andrew, 2006. Que padrões usar para medir obesidade em crianças? Disponível em: $<$ http://www.scielo.br/scielo.php?script=sci_arttext\&pid=S0021$75572006000500003 \& \operatorname{lng}=\mathrm{en} \& n r m=\mathrm{iso}>$. Acesso em: 29 de Julho de 2013. 